Received: 26 September 2017

Accepted: 7 December 2017

Published online: 21 December 2017

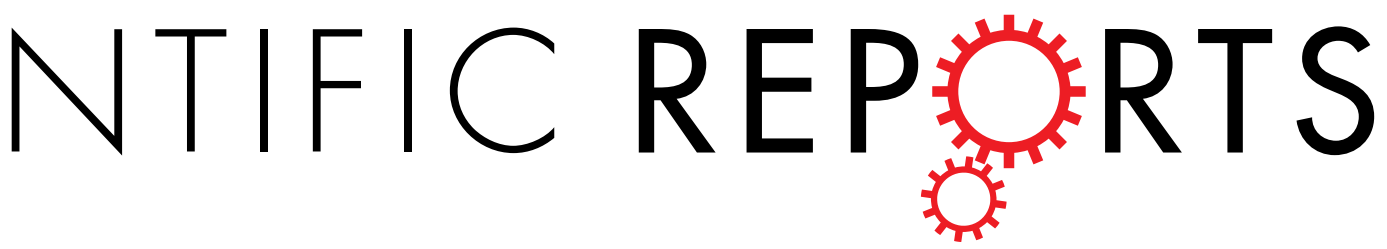

\title{
OPEN The Effect of Time, Roasting Temperature, and Grind Size on Caffeine and Chlorogenic Acid Concentrations in Cold Brew Coffee
}

\author{
Megan Fuller \& Niny Z. Rao (iD
}

The extraction kinetics and equilibrium concentrations of caffeine and 3-chlorogenic acid (3-CGA) in cold brew coffee were investigated by brewing four coffee samples (dark roast/medium grind, dark roast/ coarse grind, medium roast/medium grind, medium roast/coarse grind) using cold and hot methods. 3-CGA and caffeine were found at higher concentrations in cold brew coffee made with medium roast coffees, rather than dark roast. The grind size did not impact 3-CGA and caffeine concentrations of cold brew samples significantly, indicating that the rate determining step in extraction for these compounds did not depend on surface area. Caffeine concentrations in cold brew coarse grind samples were substantially higher than their hot brew counterparts. 3-CGA concentrations and $\mathrm{pH}$ were comparable between cold and hot brews. This work suggests that the difference in acidity of cold brew coffee is likely not due to 3-CGA or caffeine concentrations considering that most acids in coffee are highly soluble and extract quickly. It was determined that caffeine and 3-CGA concentrations reached equilibrium according to first order kinetics between 6 and 7 hours in all cold brew samples instead of 10 to 24 hours outlined in typical cold brew methods.

In 2015, domestic coffee consumption in the United States reached an estimated 1.4 billion $\mathrm{kg} / \mathrm{year}$, making it the second largest coffee market in the world after the European Union ${ }^{1}$. The majority of coffee consumed in the United States is prepared through various hot brewing methods, whereby the hot water solubilizes and extracts numerous organic compounds from the roasted coffee grounds. However, cold brew coffee preparation techniques have grown in popularity, both in at-home and consumer (or ready-to-drink, RTD) markets. Market researcher, StudyLogic, estimates that coffee shop sales of hot coffee fell $3 \%$ in 2016, while cold brewed coffee sales were up nearly $80 \%$ over the previous year's record ${ }^{2}$. Roast Magazine reports a $460 \%$ increase in retail sales of refrigerated cold brew coffee from 2015 to 2017, generating \$38 million in 2017 alone $^{3}$. In an effort to capitalize on this rapidly growing market, Dunkin' Donuts, Starbucks, and other commercial coffee vendors have invested in RTD cold brew coffee beverages and are suggesting that colder, slower brewing processes alter flavor, aroma, and bioactive compounds ${ }^{4}$. Starbucks markets that cold brew coffee is sweeter, smoother, with a more full-bodied flavor than conventionally brewed coffee ${ }^{5}$. Dunkin' Donuts claims that, "cold brew is less acidic and naturally sweeter than regular coffee, so it can easily be consumed black"6.

Cold brew coffee, not to be confused with iced coffee (which is hot brewed coffee served over ice), is prepared at room temperature $\left(20\right.$ to $25^{\circ} \mathrm{C}$ or colder) over a longer time period than traditional hot brewing methods, typically steeping times range from 8 to 24 hours $^{7-10}$. Brewing coffee is an extraction process dependent on a multitude of variables such as water volume, water temperature, diameter of the coffee grind particles, the porosity of the coffee grind matrix, the pore network between coffee grind particles, and brewing time. Temperature often significantly influences compounds aqueous solubility, so differences in brewing temperatures may result in significantly different compositions in hot brew and cold brew coffees. Additionally, the longer brewing times of cold brew coffee may affect the final composition of cold brew coffee if the diffusion of the compounds across the grind matrix is a kinetically limiting phenomenon.

An extensive body of literature exists detailing the chemistry of hot brewed coffee, including quantifying the caffeine concentration as a function of hot water brewing method ${ }^{11-14}$. Bioactive chemicals such as chlorogenic 


\begin{tabular}{|l|c|c|}
\hline Sample Name (Roast - Grind) & Grind Size (\% by mass) & Roasting Temperature \\
\hline \multirow{3}{*}{ Medium - Medium } & $3350 \mu \mathrm{m}-5.7 \%$ & \\
& $841 \mu \mathrm{m}-26.2 \%$ & $215-217^{\circ} \mathrm{C}$ \\
& $400 \mu \mathrm{m}-53.3 \%$ & \\
& $149 \mu \mathrm{m}-14.8 \%$ & \\
\hline \multirow{3}{*}{ Medium - Coarse } & $3350 \mu \mathrm{m}-0 \%$ & \\
& $841 \mu \mathrm{m}-70.6 \%$ & $215-217^{\circ} \mathrm{C}$ \\
& $400 \mu \mathrm{m}-23.1 \%$ & \\
& $149 \mu \mathrm{m}-6.3 \%$ & \\
Dark - Medium & $3350 \mu \mathrm{m}-5.2 \%$ & \\
& $841 \mu \mathrm{m}-38.1 \%$ & $223-225^{\circ} \mathrm{C}$ \\
& $400 \mu \mathrm{m}-45.4 \%$ & \\
\hline & $149 \mu \mathrm{m}-11.3 \%$ & \\
Dark - Coarse & $3350 \mu \mathrm{m}-0 \%$ & \\
& $841 \mu \mathrm{m}-77.8 \%$ & $223-225^{\circ} \mathrm{C}$ \\
& $400 \mu \mathrm{m}-17.5 \%$ & \\
& $149 \mu \mathrm{m}-4.3 \%$ & \\
&
\end{tabular}

Table 1. Summary of grind size distribution by percent mass ( $100.0 \mathrm{~g}$ of grinds used in each analysis) and roasting temperature, as reported by the coffee vendor.

acids, caffeine, and other dietary phenolic compounds that include caffeoylquinic acids, dicaffeoylquinic acids, and feruloylquinic acids ${ }^{15}$ are abundant in coffee. These chlorogenic acid compounds convey bitterness to coffee $e^{11}$ and are known to be active antioxidants that may cause health benefits in coffee drinkers ${ }^{16-18}$. The presence of these bioactive compounds in the complex chemical composition of coffee extracts have prompted numerous epidemiological studies to ascertain the degree and manner in which coffee confers health risks and/or benefits ${ }^{19}$ to the drinker. Researchers have observed both U-shaped ${ }^{19-21}$ and J-shaped ${ }^{22}$ associations between coffee consumption and risks of cardiovascular diseases. Inverse relationships have been found with coffee consumption and total mortality ${ }^{23,24}$, depression ${ }^{25}$, diabetes mellitus ${ }^{26,27}$ and certain types of cancers ${ }^{28-30}$. Work by Bakuradze, et al. showed compounds present in coffee roast products, notably 5 -caffeoylquinic acid and caffeic acid demonstrated direct antioxidant activity in HT-29 (human colon) cells ${ }^{31}$. A recent review by Naveed et al. further highlighted the therapeutic roles of chlorogenic acids in human health and called for further research in the area ${ }^{32}$. These studies often focus on analysis of green coffee beans, hot brewed coffee consumption, or make no distinction to the brewing method used. Research in hot brew coffees show that both roasting temperature ${ }^{33,34}$ and grind size $^{35,36}$ affect the extraction kinetics and maximum extractable concentration of soluble compounds from coffee grinds, specifically chlorogenic acids ${ }^{37-40}$. Increases in roasting temperatures correlate to a decrease in extractable chlorogenic acid concentrations and to an increase in caffeine concentrations $s^{33}$. However, because of the potential chemical differences between hot and cold brew coffee, it is unknown if the new popular drink will convey similar benefits to its hot brew counterpart.

Despite the increasing popularity of cold brew coffee, there is currently very little research published on the chemistry or associated health risks and/or benefits of cold brew coffee. An exhaustive literature search revealed very limited publications analyzing cold brew coffee. In 2014, Kim and Kim reported that the flavor of cold brew coffee may be more appealing to the Korean coffee consumers after 18 hours brewing time ${ }^{41}$. In 2017, Lane et al. reported that caffeine concentrations of commercially brewed cold brew coffee was $\sim 207 \mathrm{mg}$ per $12 \mathrm{fl}^{\circ} \mathrm{oz}^{42}$. A third study by Shin in 2017 reported that the polysaccharides isolated from cold brew coffee "may potentially enhance macrophage functions and the intestinal immune system" ${ }^{43}$. To date, these publications represent the majority of published research available on cold brew coffee. Commercial vendors' claims of lower acidity and other taste and chemical attributes have yet to be verified by unbiased research.

Given cold brew coffee's significant growth in the coffee market and the potential importance of coffee's bioactive compounds on human health, this research investigated the role of cold brewing methods on the kinetics and equilibrium conditions of two compounds of interest: caffeine and 3-chlorogenic acid (3-CGA). The pH of all coffees produced in this research was also measured to determine if cold brew coffee does results in a less acidic coffee beverage. This work studied the extraction kinetics of caffeine and 3-chlorogenic for both cold and hot brew methods using single origin Arabica beans grown in the Kona Region of Hawai'i in order to determine the effect water temperature and brewing time on the extraction kinetics and maximum equilibrium concentration of these two bioactive compounds. Brewing methods employed in this work mimicked standard home-brewing conditions to inform what, if any, differences consumers can expect between hot and cold brew coffees.

\section{Results}

Kinetics of cold brew coffee extraction. Four coffee samples were used in this study. See Table 1 for grind size distribution and roasting temperature characteristics for each of the samples.

The grain size distributions of the four samples show that the "medium" grind coffees had wider particle distributions, both containing about $5 \%$ of particles, by mass, that are larger than $3350 \mu \mathrm{m}$. The "coarse" grind coffees showed no $3350 \mu \mathrm{m}$ portion, and have a narrower particle distribution with more than $70 \%$ of particles, by mass, being retained on the $841 \mu \mathrm{m}$ sieve. Coffee beans are naturally porous. The pore space within each grain of coffee is considered the intragranular pores. The space between grains of coffee is referred to as the intergranular pores. Earlier studies found that particle distribution was vitally important to coffee extraction, affecting both the diffusion of compounds through intragranular pores within grinds, as well as the fluid flow between the grinds (through the intergranular pore network) $)^{14,44,45}$. The importance of both intragranular and intergranular pore space will be discussed further with respect to diffusion limiting processes of compound extraction kinetics. 

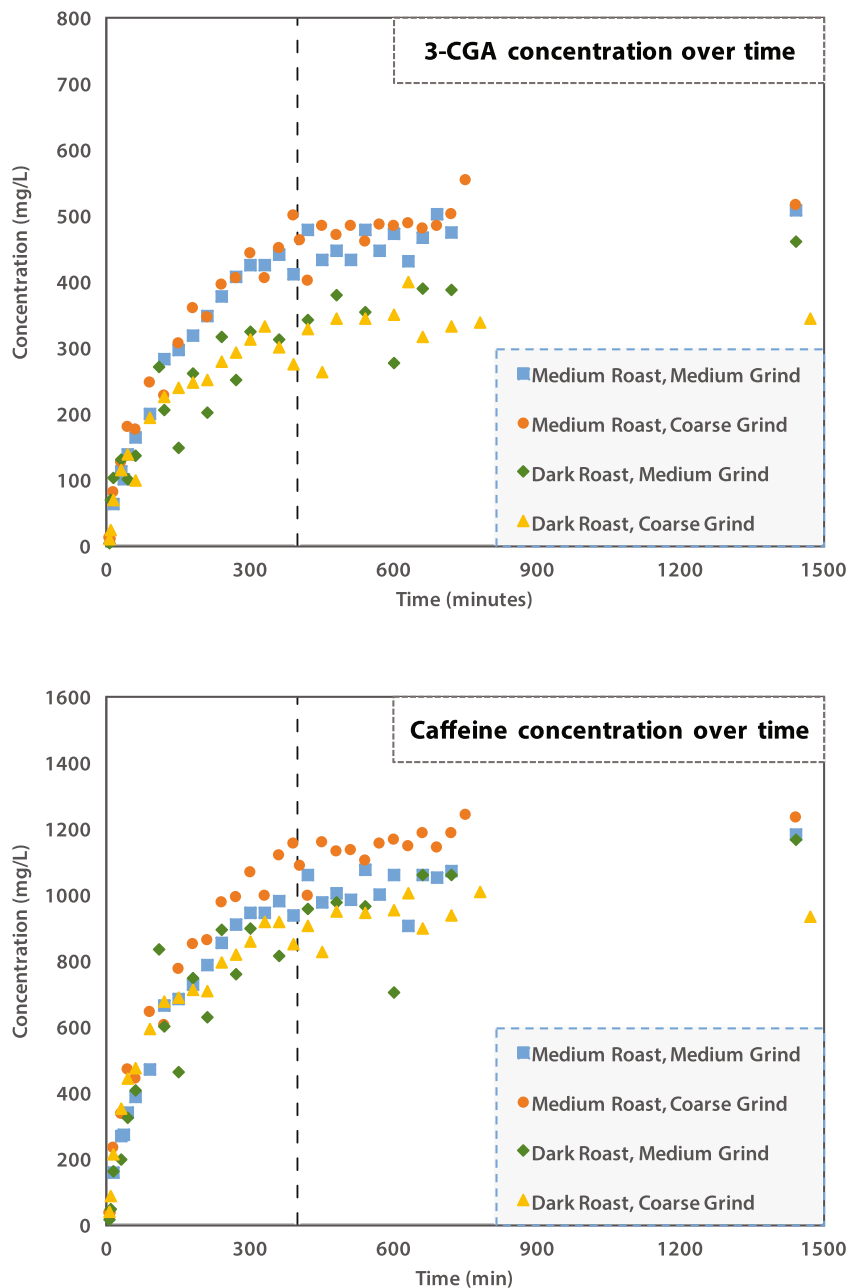

Figure 1. Concentration of 3-CGA (top) and caffeine (bottom) over time for ( $\boldsymbol{\square}$ ) medium roast - medium grind; $(\bullet)$ medium roast - coarse grind; $(\bullet)$ dark roast - medium grind, $(\mathbf{A})$ dark roast - coarse grind. The vertical line at 400 minutes represents the establishment of steady-state concentration for both 3-CGA and caffeine extractions.

3-CGA. The compound, 3-CGA is freely soluble in water at room temperature ${ }^{46}$. Initial 3-CGA concentration increased rapidly over the first 180 minutes and slowed until reaching equilibrium at approximately 400 minutes for all coffee roasts and grinds (see Fig. 1). Moroney et al ${ }^{45}$ attributed the initial fast extraction of soluble coffee compounds to the extraction of compounds from the surface and near-surface volume of the solid coffee grind matrix. The slower, longer time-scale extraction of additional CGA concentration, post 180 minutes, is likely due to the mass transfer of the compound through intra-grain pores into intergrain pores, and ultimately into the bulk

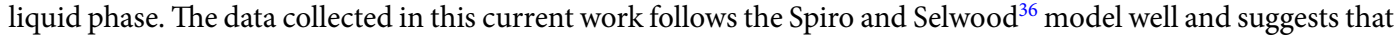
cold brew processes of 3-CGA extraction are governed by first-order kinetics. A sample kinetic plot of $\ln \left(\mathrm{C}_{\infty} /\right.$ $\left[\mathrm{C}_{\infty}-\mathrm{C}\right]$ ) for 3-CGA versus time is shown in Fig. 2 where $\mathrm{C}_{\infty}$ is the equilibrium concentration of 3-CGA and $\mathrm{C}$ is the concentration of 3-CGA at time $t$. Several sources ${ }^{7-10}$ providing brewing instructions for cold brew coffee recommend prolonged brewing times upwards of 12 to 24 hours. Any water/grind interaction longer than $400 \mathrm{~min}-$ utes (6.7 hours) did not result in additional significant extraction of 3-CGA. The mean concentrations of 3-CGA at 400 and 1400 minutes were within one standard deviation of each other (see Table 1). These data suggest 3-CGA concentrations are influenced by roasting temperature, but not grind size. Blumberg et al. ${ }^{11}$ determined that increased roasting temperatures resulted in degradation of chlorogenic acid precursors and lower extractable total chlorogenic acid concentrations. The same study also observed that chlorogenic acids extracted quickly from coffee grinds, while 4 -vinylcatechol oligomers showed strong retention to the coffee grinds ${ }^{11}$. The longer steeping times associated with cold brew coffee may result in increased extraction of these catechol oligomers, which are characterized by harsh bitter-tasting properties. Over-brewing cold brew coffee may result in unpalatable extracts due to these and other relatively slow-extracting compounds.

Caffeine. Caffeine, unlike 3-CGA, has a limited solubility at room temperature of $16 \mathrm{mg} / \mathrm{mL}^{46}$. However, all four coffees analyzed showed comparable extraction kinetics to those of 3-CGA. In all coffees sampled, fast initial extraction was seen over the first 180 minutes, with a slower rate of extraction after 180 minutes. Similar to 


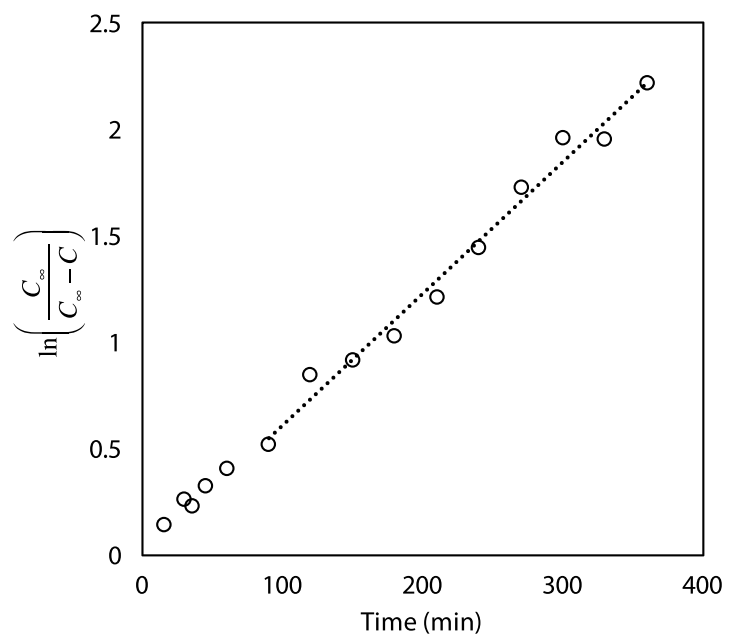

Figure 2. First-order plot for the extraction of 3-CGA from medium grind - medium roast coffee particles at $23.5^{\circ} \mathrm{C} . \mathrm{R}^{2}=0.983$.

3-CGA, caffeine also reaches nearly steady-state concentrations after 400 minutes (see Fig. 1). As with 3-CGA, all samples followed a first-order kinetic model. Spiro and Selwood ${ }^{36}$ offered a thorough mathematical model for the kinetics of caffeine extraction at room temperature, and found that the diffusion of caffeine through the intragranular pore space to be the rate limiting step in the extraction process. This analysis concluded that extraction times greater than 400 minutes do little to increase the caffeine concentration of the resulting coffee. Moreover, caffeine concentrations do not demonstrate the same sensitivity to roasting temperatures as 3-CGA, and all coffee roasts and grinds were found to have comparable caffeine concentrations at equilibrium, with the exception of the dark roast - coarse grind coffee. The relatively high standard deviations are suspected to be caused by the heterogeneous grind size distributions from the commercially sourced beans. As the packaging was handled, settling of finer particles may have caused inter-sample variability.

pH. Work by Andueza et al. ${ }^{47}$ and Gloess et al. ${ }^{48}$ both report there is no correlation between $\mathrm{pH}$ and perceived acidity in the flavor of coffees. However, commercial coffee vendors continue to relate acidity to coffee taste when marketing coffee to consumers. The $\mathrm{pH}$ of coffee studied in this work ranged from 5.40 to 5.63. Moon et al. ${ }^{15}$ observed a correlation between total CGA concentrations and $\mathrm{pH}$ of coffee extracts. However, data collected in this work did not provide enough evidence to support the claim by Moon et al. ${ }^{15}$ that coffee samples containing high concentration of 3-CGA tend to have high acidity or low $\mathrm{pH}$.

Comparison of hot brew and cold brew coffee. There is a common marketing message that cold brew coffee is fundamentally different than hot brew coffee. This may be attributed to acidity and/or caffeine concentration $^{49,50}$. This work compared the same water-to-coffee ratio using cold brew and hot brew extraction processes to identify any differences between the two methods with respect to 3-CGA and caffeine concentrations. In the coffee extraction process, Moroney et al. ${ }^{35}$ described two different extraction mechanisms that function on different timescales. The fast extraction from the surface and near-surface matrix happens much more rapidly than the diffusion of compounds through the intragranular pore network to the grain surface. Because the time periods for hot brew and cold brew are drastically different, 6 minutes vs. 1440 minutes respectively, the intragranular diffusion may limit the extractable concentration of soluble coffee compounds in the hot brew, as compared to the cold brew.

3-CGA. In Fig. 3, the cold brew extraction of caffeine and 3-CGA are shown for each of the four coffee samples, with the hot brew concentrations indicated by horizontal lines. Table 2 shows the equilibrium concentrations of 3-CGA for the hot and cold brew coffees along with the $\mathrm{pH}$. In both hot and cold brew extractions, all samples show comparable average 3-CGA concentrations and $\mathrm{pH}$, regardless of water temperature. The CGA molecule is not seen to be limited by the intragranular pore diffusion processes, as observed with caffeine extraction. CGA is freely soluble in water, and this facilitates its extraction at both low and high temperatures. While grain size did not impact the magnitude of 3-CGA concentrations, roasting temperature of the beans did show a noticeable effect in both cold and hot experiments. In both hot and cold brew extractions, CGA was found in higher concentrations in medium roasts than in darks roasts, supporting the work of Trugo and Macrae ${ }^{37}$ that suggests that higher roasting temperatures decomposes CGA and results in lower extraction concentrations.

Caffeine. Coarse grain samples, both medium and dark roast, showed a considerable deviation in caffeine concentrations between hot and cold brew extractions (Table 3). In both samples, the cold brew coffee was found to have the higher concentration of caffeine. Medium grain samples also showed higher concentrations of caffeine in cold brew extraction, however, the difference was not statistically significant. This result suggests that the medium grind blends, in both hot and cold extraction, experienced nearly complete extraction during their respective steeping times. The hot brew extraction saturated the intra- and intergranular pores and facilitated fast 

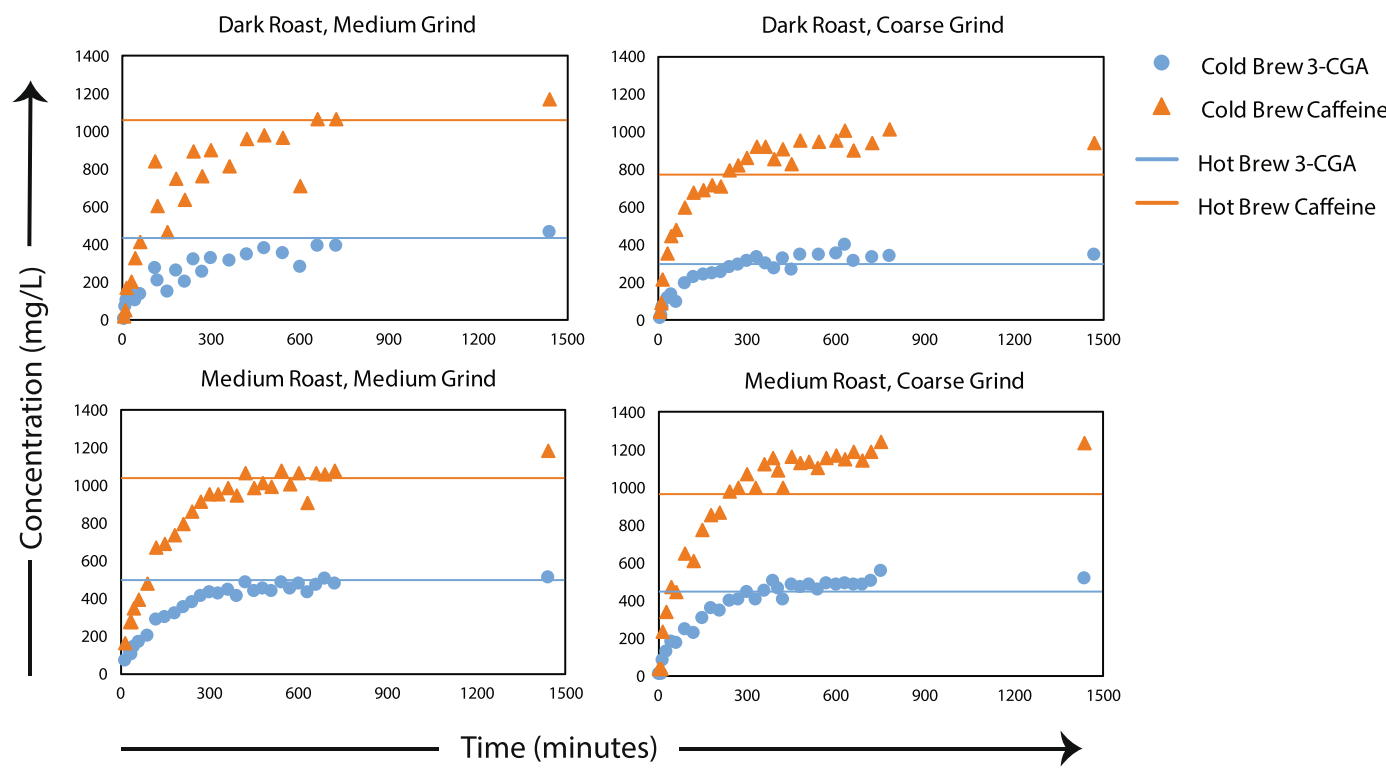

Figure 3. Caffeine $(\boldsymbol{\Delta})$ and 3-CGA $(\bullet)$ concentration as a function of time for each of the four coffee samples. Horizontal lines represent each coffee type's hot water concentration for caffeine and 3-CGA. Error bars represent the range for each measurement.

\begin{tabular}{|c|c|c|c|c|c|c|}
\hline \multirow[b]{2}{*}{$\begin{array}{l}\text { Coffee Sample } \\
\text { (Roast - Grind) }\end{array}$} & \multicolumn{3}{|c|}{$400 \mathrm{~min}$} & \multicolumn{3}{|c|}{$1440 \mathrm{~min}$} \\
\hline & $\begin{array}{c}\text { 3-CGA } \\
\text { Concentration }(\mathrm{mg} / \mathrm{L})\end{array}$ & $\begin{array}{c}\text { Caffeine } \\
\text { Concentration }(\mathrm{mg} / \mathrm{L})\end{array}$ & $\mathrm{pH}$ & $\begin{array}{c}\text { 3-CGA } \\
\text { Concentration }(\mathrm{mg} / \mathrm{L})\end{array}$ & \begin{tabular}{|c|} 
Caffeine \\
Concentration $(\mathrm{mg} / \mathrm{L})$
\end{tabular} & $\mathrm{pH}$ \\
\hline Medium - Medium & $480 \pm 60$ & $1060 \pm 60$ & $5.61 \pm 0.01$ & $510 \pm 20$ & $1180 \pm 90$ & $5.54 \pm 0.02$ \\
\hline Medium - Coarse & $490 \pm 30$ & $1130 \pm 50$ & $5.47 \pm 0.01$ & $520 \pm 40$ & $1230 \pm 60$ & $5.40 \pm 0.01$ \\
\hline Dark - Medium & $380 \pm 10$ & $970 \pm 60$ & $5.63 \pm 0.01$ & $390 \pm 10$ & $1080 \pm 70$ & $5.53 \pm 0.01$ \\
\hline Dark - Coarse & $330 \pm 50$ & $930 \pm 40$ & $5.51 \pm 0.02$ & $360 \pm 20$ & $990 \pm 30$ & $5.41 \pm 0.02$ \\
\hline
\end{tabular}

Table 2. Concentration of 3-CGA and caffeine and $\mathrm{pH}$ of each cold brew coffee sample after 400 minutes and 1440 minutes of brewing time (Mean $\pm 95 \%$ Confidence Interval, $n=6$ ).

\begin{tabular}{|c|c|c|c|c|c|c|}
\hline \multirow[b]{2}{*}{$\begin{array}{l}\text { Coffee Sample } \\
\text { (Roast - Grind) }\end{array}$} & \multicolumn{2}{|c|}{ 3-CGA Concentration $(\mathrm{mg} / \mathrm{L})$} & \multicolumn{2}{|c|}{ Caffeine Concentration (mg/L) } & \multicolumn{2}{|c|}{$\mathrm{pH}$} \\
\hline & $\begin{array}{l}\text { Cold Brew } \\
\text { Method }\end{array}$ & $\begin{array}{l}\text { Hot Brew } \\
\text { Method }\end{array}$ & $\begin{array}{l}\text { Cold Brew } \\
\text { Method }\end{array}$ & $\begin{array}{l}\text { Hot Brew } \\
\text { Method }\end{array}$ & $\begin{array}{l}\text { Cold Brew } \\
\text { Method }\end{array}$ & $\begin{array}{l}\text { Hot Brew } \\
\text { Method }\end{array}$ \\
\hline Medium - Medium & $510 \pm 20$ & $510 \pm 30$ & $1180 \pm 90$ & $1040 \pm 70$ & $5.54 \pm 0.02$ & $5.41 \pm 0.02$ \\
\hline Medium - Coarse & $520 \pm 40$ & $460 \pm 40$ & $1230 \pm 60$ & $970 \pm 70$ & $5.40 \pm 0.01$ & $5.35 \pm 0.03$ \\
\hline Dark - Medium & $390 \pm 10$ & $430 \pm 30$ & $1080 \pm 70$ & $1060 \pm 70$ & $5.53 \pm 0.01$ & $5.61 \pm 0.02$ \\
\hline Dark - Coarse & $360 \pm 20$ & $340 \pm 10$ & $990 \pm 30$ & $840 \pm 10$ & $5.41 \pm 0.02$ & $5.48 \pm 0.02$ \\
\hline
\end{tabular}

Table 3. Comparison of equilibrium 3-CGA and caffeine concentrations (after $1440 \mathrm{~min}$ ) extracted using cold and hot brew method along with $\mathrm{pH}$ (mean $\pm 95 \%$ Confidence Interval, $n=6$ ).

diffusion (6-minute steeping times) of caffeine across the solid matrix to generate a bulk liquid phase with nearly the same concentration of caffeine as the cold brew coffee generated in 400 minutes. Coarse grain samples, with their higher relative proportion of particles in the $841 \mu \mathrm{m}$ range, did not reach similar steady-state concentrations in both hot and cold brews. The faster, hot water extraction was diffusion limited, and likely did not allow the full extraction of caffeine across the larger radius particles. The longer brewing times for the cold brew samples resulted in greater caffeine extraction, allowing time for completion of the rate-limiting mass transfer step in the extraction process.

Role of Grind Size and Roasting Temp in Cold Brew Coffee. Further analysis of the data indicates that the observed CGA and caffeine concentration differences between medium roast and dark roast are, in general, substantial. Both CGA and caffeine showed higher concentration in medium roast samples. Our data is in support of the works of Trugo, et al. ${ }^{37}$ and Hečimović, et al. ${ }^{51}$, both suggest that higher roasting temperatures decrease the concentration of CGA and caffeine. The only exception is the observed difference in concentration of caffeine 

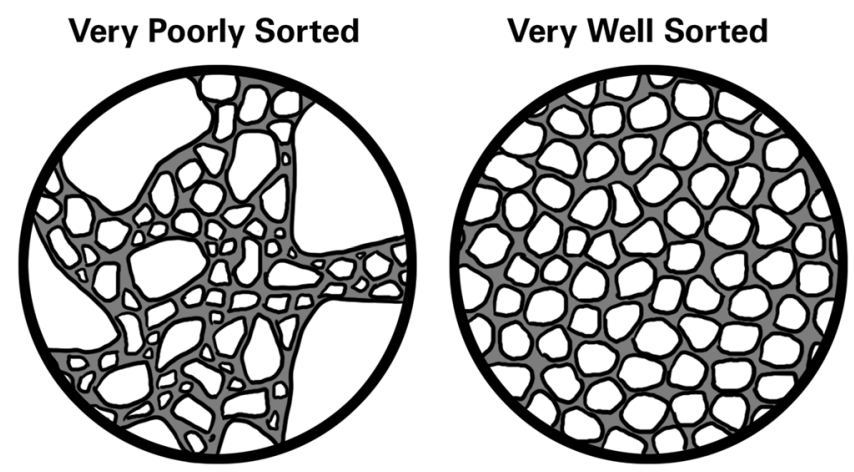

Figure 4. The graphical image of sorting effects on pore connectivity in coffee matrix beds. Grain size distribution influences both the intragranular diffusion process, as larger grains have greater diffusion distances, and the intergranular pore network, as poorly sorted grain beds have more tortuous pore networks.

when comparing medium roast - medium grind and dark roast - medium grind samples. Although the medium roast samples showed higher concentration of caffeine than dark roast samples, the observed difference in concentration is insignificant due to large variations in the measurements.

\section{Discussion and Conclusional Remarks}

This work establishes that brewing times near 400 minutes are adequate to extract the majority of available caffeine and 3-CGA in medium and dark roast beans prepared at medium and coarse grinds. Moreover, coarse grain samples, both medium and dark roast, showed a substantial increase in caffeine concentrations than their hot brew counterparts. No significant differences were seen in CGA concentrations between cold and hot brews. Furthermore, the $\mathrm{pH}$ between cold and hot brews were comparable. This work suggests that any claims made by coffee vendors about the difference in acidity or taste of cold brew coffee is not due to variations in 3-CGA concentrations. The results of this study validate earlier models proposed by by Moroney et al ${ }^{35,45}$ and Spiro and Seldwood ${ }^{52}$ and extend their findings into cold brew extraction over long time periods.

Furthermore, our analysis indicates that the grind size does not have significant impact the observed equilibrium concentrations for both CGA and caffeine. When comparing samples with the same roasting temperatures, the observed differences in concentrations are largely within one standard deviation from another. Mathematical modeling of coffee extraction proposed by Moroney, et al. ${ }^{35,45}$ suggested that the diffusion of coffee from the intragranular pores to the intergranular pores is the rate limiting process. Thus, it takes longer for the extraction process to reach equilibrium as the grind size increases. However, in the cold brew process, the extraction time frame is on the order of hours instead of seconds. Such long extraction time scales allow for the slow diffusion from intragranular to intergranular pores, so this is not a rate determining process in cold brew methodologies. We have noted that grind size was not well controlled in this study, as this work used commercially available coffee without any size separation prior to extraction. Future work will differentiate coffee grinds by particle size to further quantify the role of grind size in cold brew coffee. Figure 4 shows a graphical representation of a poorly sorted and a well sorted coffee grind. Grind size and grind sorting are both important parameters controlling inter- and intragranular diffusion.

\section{Materials and Methods}

Materials. Coffee from the Kona region of Hawai'i was sourced from Kona Joe Coffee, LLC (Kealakekua, HI). This single origin Arabica (Kona Typica) coffee was obtained roasted and ground from Kona Joe Coffee following their standard preparation processes. Four coffee types were used in this study. Two roasting temperatures, medium and dark, prepared at two grind sizes, medium and coarse were selected for this work. Both roasting and grinding were done by Kona Joe Coffee. Medium roast beans were roasted at $215^{\circ} \mathrm{C}$ to $217^{\circ} \mathrm{C}$, Dark roast beans were roasted at $223^{\circ} \mathrm{C}$ to $225^{\circ} \mathrm{C}$. The beans were ground using a Mahlkönig DK-15 industrial grinder.

Standard stock solutions of $400 \mathrm{mg} / \mathrm{L}$ caffeine and 3-CGA were made daily and diluted to establish calibration curves for coffee analysis. Both purchased from Sigma Aldrich (Milwaukee, WI). HPLC grade methanol was obtained from Fisher Scientific (Nazareth, PA). Phosphoric acid (85\% wt.) was obtained from Sigma Aldrich (Milwaukee, WI) and diluted to $2.0 \mathrm{mM}$ concentration using DI water. Filtered municipal tap water used in this study. Analysis of this water, completed by Penn State University's Agricultural Analytical Services Laboratory found the water to have a total hardness of $174 \mathrm{mg} / \mathrm{L}$ and a $\mathrm{pH}$ of 7.5 .

Methods. Particle size distribution. The particle size distributions of each grind were determined according to ASTM C136/C136M-14 Standard Test Method for Sieve Analysis for Fine and Coarse Aggregates procedure. Samples of $100.0 \mathrm{~g}$ of coffee grinds were added to a sieve stack including sieve sizes \#20 (0.841 mm mesh opening), \#40 (0.420 mm mesh opening), \#100 mesh (0.149 mm mesh opening), and pan, to generate grain size distributions for each coffee used in this study.

Cold brew experiments. The cold brewing process was carried out at room temperature (ranging from $21^{\circ} \mathrm{C}$ to $25^{\circ} \mathrm{C}$ over the experimental period) adapted from a home-brewing recipe suggested by The New York Times' 
Cooking website ${ }^{9}$. A sample of $35.0 \mathrm{~g}$ of coffee was placed in $350 \mathrm{~mL}$ of carbon-filtered municipal water. The coffee was contained in T-Sac ${ }^{\mathrm{TM}}$ tea filter bag (size 4) and placed in a 32-ounce Mason jar fitted with a screw-top lid. The filter bag was used to reduce grind loss during sampling and ensure grinds remained submerged during steeping. The coffee/water mixture was sampled every 15 minutes for the first hour, then every 30 minutes until hour 7, and then once an hour until hour 12, a final sample was taken at 24 hours. Samples collected after the first hour were diluted (1:4) with DI water and filtered using HT Tuffryn (Pall) $25 \mathrm{~mm}$ diameter, $0.2 \mu \mathrm{m}$ pore size membranes. Fresh water was added to replace the volume sampled to maintain constant volume. This introduced a small dilution effect in the resulting solution. Additionally, even with the closed system, there was inevitably evaporation over the 24 hour testing period. Coffee received from Kona Joe Coffee was not processed in any way prior to use, to best match home-brewing conditions. Data presented are an average of triplicate experiments analyzed in duplicates $(n=6)$.

Hot brew experiments. Hot brew extraction was conducted using the same coffee to water ratio as was used in the cold brew method. The water was heated to $98^{\circ} \mathrm{C}$ and added to coffee grounds in a traditional French press carafe. The water and grounds were allowed to sit for 6 minutes before the filter was depressed and the coffee decanted. Since additional experiments showed that longer mixing times did not result in additional caffeine or 3-CGA extraction, 6-minute extraction times were used for all hot brew experiments. Two samples were taken from each hot brew and each experiment was performed in triplicate $(n=6)$.

Caffeine and 3-CGA measurement. Caffeine and 3-CGA were measured in both standard solutions and coffee extracts using an adapted methodology reported in GL Sciences Technical Note No. $67^{53}$. An Agilent 1200 Series high performance liquid chromatography system (HPLC) was fitted with a Supelco $5 \mu \mathrm{m}$ column $(15 \mathrm{~cm} \times 4.6 \mathrm{~cm})$ (Supleco, Bellefonte, $\mathrm{PA}$ ) run at $40.0^{\circ} \mathrm{C}$ with a mobile phase mixture of $75 \%$ mobile phase A and $25 \%$ mobile phase B (A: 95\% $2.0 \mathrm{mM}$ phosphoric acid and 5\% methanol; B: 95\% methanol and 5\% $2.0 \mathrm{mM}$ phosphoric acid). The flow rate was $1.0 \mathrm{~mL} / \mathrm{min}$ with an injection volume of $10.0 \mu \mathrm{L}$. Caffeine and 3-CGA were detected using a diode array detector at $280 \mathrm{~nm}$ and $325 \mathrm{~nm}$ respectively.

pH measurements. The $\mathrm{pH}$ of each brewed coffee sample was measured with a Mettler Toledo FiveEasy ${ }^{\mathrm{TM}}$ F20 benchtop $\mathrm{pH} / \mathrm{mV}$ meter.

Statistical Analysis. Two-tailed t-test and ANOVA were employed for determination of similarities in equilibrium concentrations of 3-CGA and caffeine with consideration of the roast, grind size, and brewing method. The output of the statistical analysis is included in the supporting information.

Data Availability. All data generated or analyzed during this study are included in this published article (and its Supplementary Information files).

\section{References}

1. Loftfield, E. et al. Coffee Drinking Is Widespread in the United States, but Usual Intake Varies by Key Demographic and Lifestyle Factors. J. Nutr. 146, 1762-1768 (2016).

2. Perez, M. Cold Brew Might Save the Coffee Market. Bloomberg Markets (2017). Available at: https://www.bloomberg.com/news/ articles/2017-07-19/hot-sales-of-cold-brew-might-just-be-what-coffee-market-needs. (Accessed: 11th November 2017).

3. Brown, N. New US Coffee Shop Growth Slows as RTD and Cold Brew Accelerate, According to Mintel. Roast Magazine (2017). Available at: https://dailycoffeenews.com/2017/10/03/new-us-coffee-shop-growth-slows-as-rtd-and-cold-brew-accelerateaccording-to-mintel/. (Accessed: 11 th November 2017).

4. Website. Starbucks Cold Brew Coffee Available at: http://lp.starbucks.com/coldbrew. (Accessed: 8th May 2017).

5. Starbucks ${ }^{\circledR}$ Cold Brew Coffee. Available at: http://lp.starbucks.com/coldbrew. (Accessed: 26th July 2017).

6. cold-brew | Dunkin' Donuts. Available at: https://www.dunkindonuts.com/en/food-drinks/iced-drinks/cold-brew. (Accessed: 26th July 2017).

7. Toddy Instruction Manual and Guide | Toddy Cold Brew. Available at: https://toddycafe.com/cold-brew/instruction-manual. (Accessed: 26th July 2017).

8. Food. How to Make Cold-Brewed Coffee. Food52 (2017). Available at: https://food52.com/blog/7317-how-to-make-cold-brewedcoffee. (Accessed: 26th July 2017).

9. Times, T. N. Y. Cold-Brewed Iced Coffee Recipe. NYT Cooking Available at: https://cooking.nytimes.com/recipes/1017355-coldbrewed-iced-coffee. (Accessed: 26th July 2017).

10. 1912 Pike.com Story, A. How To Make Cold Brew Coffee at Home. Starbucks Store Available at: http://store.starbucks.com/blog/ how-to-cold-brew-coffee-at-home. (Accessed: 26th July 2017).

11. Blumberg, S., Frank, O. \& Hofmann, T. Quantitative studies on the influence of the bean roasting parameters and hot water percolation on the concentrations of bitter compounds in coffee brew. J. Agric. Food Chem. 58, 3720-3728 (2010).

12. Blank, I., Sen, A. \& Grosch, W. Potent odorants of the roasted powder and brew of Arabica coffee. Zeitschrift für LebensmittelUntersuchung und -Forschung 195, 239-245 (1992).

13. Shibamoto, T. et al. Application of HPLC for evaluation of coffee flavor quality. In The Quality of Foods and Beverages 311-334 (1981).

14. Bell, L. N., Wetzel, C. R. \& Grand, A. N. Caffeine content in coffee as influenced by grinding and brewing techniques. Food Res. Int. 29, 785-789 (1996).

15. Moon, J.-K., Yoo, H. S. \& Shibamoto, T. Role of roasting conditions in the level of chlorogenic acid content in coffee beans: correlation with coffee acidity. J. Agric. Food Chem. 57, 5365-5369 (2009).

16. Niggeweg, R., Michael, A. J. \& Martin, C. Engineering plants with increased levels of the antioxidant chlorogenic acid. Nat. Biotechnol. 22, 746-754 (2004).

17. Sato, Y. et al. In vitro and in vivo antioxidant properties of chlorogenic acid and caffeic acid. Int. J. Pharm. 403, 136-138 (2011).

18. del Castillo, M. D., Ames, J. M. \& Gordon, M. H. Effect of roasting on the antioxidant activity of coffee brews. J. Agric. Food Chem. 50, 3698-3703 (2002).

19. Chu, Y.-F. Coffee: Emerging Health Effects and Disease Prevention. (John Wiley \& Sons, 2012). 
20. Nordestgaard, A. T. \& Nordestgaard, B. G. Coffee intake, cardiovascular disease and all-cause mortality: observational and Mendelian randomization analyses in 95 000-223 000 individuals. Int. J. Epidemiol. https://doi.org/10.1093/ije/dyw325 (2016).

21. Moghadasian, M. H. \& Eskin, N. A. M. Functional Foods and Cardiovascular Disease. (CRC Press, 2012).

22. Kouli, G.-M. et al. J-shaped relationship between habitual coffee consumption and 10-year (2002-2012) cardiovascular disease incidence: the ATTICA study. Eur. J. Nutr (2017).

23. Malerba, S. et al. A meta-analysis of prospective studies of coffee consumption and mortality for all causes, cancers and cardiovascular diseases. Eur. J. Epidemiol. 28, 527-539 (2013).

24. Preedy, V. R. Coffee in Health and Disease Prevention. (Academic Press, 2014).

25. Wang, L., Shen, X., Wu, Y. \& Zhang, D. Coffee and caffeine consumption and depression: A meta-analysis of observational studies. Aust. N. Z. J. Psychiatry 50, 228-242 (2016).

26. Shang, F., Li, X. \& Jiang, X. Coffee consumption and risk of the metabolic syndrome: A meta-analysis. Diabetes Metab. 42, 80-87 (2016).

27. Chrysant, S. G. The impact of coffee consumption on blood pressure, cardiovascular disease and diabetes mellitus. Expert Rev. Cardiovasc. Ther. 15, 151-156 (2017).

28. Sang, L.-X., Chang, B., Li, X.-H. \& Jiang, M. Consumption of coffee associated with reduced risk of liver cancer: a meta-analysis. BMC Gastroenterol. 13, 34 (2013).

29. Li, G., Ma, D., Zhang, Y., Zheng, W. \& Wang, P. Coffee consumption and risk of colorectal cancer: a meta-analysis of observational studies. Public Health Nutr. 16, 346-357 (2013).

30. Je, Y. \& Giovannucci, E. Coffee consumption and risk of endometrial cancer: findings from a large up-to-date meta-analysis. Int. J. Cancer 131, 1700-1710 (2012).

31. Bakuradze, T. et al. Antioxidant effectiveness of coffee extracts and selected constituents in cell-free systems and human colon cell lines. Mol. Nutr. Food Res. 54, 1734-1743 (2010).

32. Naveed, M. et al. Chlorogenic acid (CGA): A pharmacological review and call for further research. Biomed. Pharmacother. 97, 67-74 (2017).

33. Lang, R. et al. Quantitative studies on roast kinetics for bioactives in coffee. J. Agric. Food Chem. 61, 12123-12128 (2013).

34. Spiro, M. \& Hunter, J. E. The kinetics and mechanism of caffeine infusion from coffee: The effect of roasting. J. Sci. Food Agric. 36, 871-876 (1985)

35. Moroney, K. M., Lee, W. T., O’Brien, S. B. G., Suijver, F. \& Marra, J. Coffee extraction kinetics in a well mixed system. J. Math. Ind. 7 (2016).

36. Spiro, M. \& Selwood, R. M. The kinetics and mechanism of caffeine infusion from coffee: The effect of particle size. J. Sci. Food Agric. 35, 915-924 (1984).

37. Trugo, L. C. \& Macrae, R. A study of the effect of roasting on the chlorogenic acid composition of coffee using HPLC. Food Chem. 15, 219-227 (1984).

38. Farah, A., de Paulis, T., Trugo, L. C. \& Martin, P. R. Effect of Roasting on the Formation of Chlorogenic Acid Lactones in Coffee. J. Agric. Food Chem. 53, 1505-1513 (2005).

39. Fujioka, K. \& Shibamoto, T. Chlorogenic acid and caffeine contents in various commercial brewed coffees. Food Chem. 106, 217-221 (2008).

40. Casal, S., Beatriz Oliveira, M. \& Ferreira, M. A. HPLC/diode-array applied to the thermal degradation of trigonelline, nicotinic acid and caffeine in coffee. Food Chem. 68, 481-485 (2000).

41. Kim, A. R. \& Kim, J. S. Flavor Contributing Nonvolatile Chemical and Sensory Characterization of Cold Water Extraction-based Coffee by Different ExtractionMethods (Dripping vs Steeping) and Time. Journal of The Korea Society for Coffee Industry 3, 1-9 (2014).

42. Lane, S., Palmer, J., Christie, B., Ehlting, J. \& Le, C. Can Cold Brew Coffee Be Convenient? A Pilot Study For Caffeine Content in Cold Brew Coffee Concentrate Using High Performance Liquid Chromatography. The Arbutus Review 8, 15-23 (2017).

43. Shin, K.-S. The Chemical Characteristics and Immune-Modulating Activity of Polysaccharides Isolated from Cold-Brew Coffee. Prev Nutr Food Sci 22, 100-106 (2017).

44. Uman, E. et al. The effect of bean origin and temperature on grinding roasted coffee. Sci. Rep. 6, 24483 (2016).

45. Moroney, K. M., Lee, W. T., O’Brien, S. B. G., Suijver, F. \& Marra, J. Modelling of coffee extraction during brewing using multiscale methods: An experimentally validated model. Chem. Eng. Sci. 137, 216-234 (2015).

46. Budavari, S., O'Neil, M., Smith, A., Heckelman, P. \& Obenchain, J. The Merck Index, Print Version, Twelfth Edition. (CRC Press, 1996).

47. Andueza, S., Vila, M. A., de Peña, M. P. \& Cid, C. Influence of coffee/water ratio on the final quality of espresso coffee. J. Sci. Food Agric. 87, 586-592 (2007).

48. Gloess, A. N. et al. Comparison of nine common coffee extraction methods: instrumental and sensory analysis. Eur. Food Res. Technol. 236, 607-627 (2013)

49. Calderone, J. Website. Chemistry explains why cold brew coffee tastes better than hot (2015). Available at: http://www.businessinsider. com/cold-brew-coffee-taste-chemistry-science-solubles-volatile-2015-8. (Accessed: 26th July 2017).

50. Murray, R. Website. What is cold brew coffee and why is everyone - including Starbucks - into it? (2015). Available at: http://www. today.com/food/what-cold-brew-coffee-why-everyone-including-starbucks-it-t9481. (Accessed: 26th July 2017).

51. Hečimović, I., Belščak-Cvitanović, A., Horžić, D. \& Komes, D. Comparative study of polyphenols and caffeine in different coffee varieties affected by the degree of roasting. Food Chem. 129, 991-1000 (2011).

52. Spiro, M. \& Selwood, R. M. The kinetics and mechanism of caffeine infusion from coffee: The effect of particle size. J. Sci. Food Agric. 35, 915-924 (1984).

53. Analysis of Chlorogenic Acid in Coffee by HPLC. Available at: https://www.glsciences.com/pdf/technicalnote_lc/067.pdf. (Accessed: 26th July 2017).

\section{Acknowledgements}

The authors would like to thank Nicolas A. Parenti, Samantha Ryder, Nelly Tchato Setchie, and Maria Latorre Socas for their contribution in data collection. This work would not have been possible without their oversight of sample collection, HPLC analysis, and $\mathrm{pH}$ measurement. The authors would like to thank the generous funding support provided by the Eileen Martinson '86 Fund for the Undergraduate Capstone Experience at the Thomas Jefferson University East Falls Campus. The authors would also like to thank Dr. Joseph Alban and Mr. Bruno Prota at Kona Joe Coffee for their insightful suggestions and discussions.

\section{Author Contributions}

M.F. contributed to the experimental design, execution. N.Z.R. conceived of the study, aided in experimental design and execution. M.F. and N.Z.R. contributed equally to all versions of the manuscript. 


\section{Additional Information}

Supplementary information accompanies this paper at https://doi.org/10.1038/s41598-017-18247-4.

Competing Interests: The authors declare that they have no competing interests.

Publisher's note: Springer Nature remains neutral with regard to jurisdictional claims in published maps and institutional affiliations.

(c) (i) Open Access This article is licensed under a Creative Commons Attribution 4.0 International License, which permits use, sharing, adaptation, distribution and reproduction in any medium or format, as long as you give appropriate credit to the original author(s) and the source, provide a link to the Creative Commons license, and indicate if changes were made. The images or other third party material in this article are included in the article's Creative Commons license, unless indicated otherwise in a credit line to the material. If material is not included in the article's Creative Commons license and your intended use is not permitted by statutory regulation or exceeds the permitted use, you will need to obtain permission directly from the copyright holder. To view a copy of this license, visit http://creativecommons.org/licenses/by/4.0/.

(C) The Author(s) 2017 